Schweiz. Gastroenterol. 2021 · 2:119-127 https://doi.org/10.1007/s43472-021-00058-8

Angenommen: 5. November 2021

Online publiziert: 3. Dezember 2021

( $\odot$ The Author(s), under exclusive licence to Springer-Verlag GmbH Austria, ein Teil von Springer Nature 2021

\section{Einleitung}

Die Leberzirrhose ist das Endstadium chronischer Leberkrankheiten. In der Schweiz sind die häufigsten Ursachen für eine Leberzirrhose Alkoholüberkonsum, die nichtalkoholische Steatohepatitis, eine chronische Hepatitis-B- oder Hepatitis-C-Virusinfektion. Seltenere Ursachen sind die Hämochromatose, Autoimmunhepatitis, cholestatische Lebererkrankungen wie die primär biliäre Cholangitis und primär sklerosierende Cholangitis, der Morbus Wilson, eine kongenitale Leberfibrose und medikamentös-toxische Ursachen. Schätzungsweise 50.000 Personen sind schweizweit von einer Leberzirrhose betroffen [1]. Die Leberzirrhose ist mit einer vermehrten Morbidität und Mortalität sowie einer reduzierten Lebensqualität assoziiert. Pathophysiologisch führt die progrediente Fibrosierung des Lebergewebes zur Entstehung einer portalen Hypertonie, welche für viele Komplikationen wie Varizenblutungen, Aszites, hepatische Enzephalopathie und das hepatorenale Syndrom verantwortlich ist. Daneben sind Patienten mit Leberzirrhose gefährdet, an einem hepatozellulären oder cholangiozellulären Karzinom zu erkranken. Für den Kliniker ist die Betreuung von Patienten mit Leberzirrhose anspruchsvoll. Neben der Behandlung der Grunderkrankung gilt es, Komplikationen zu verhindern oder frühzeitig zu erkennen. Bei Dekompensation und zunehmender Leberinsuffizienz sollen geeignete Patienten für eine Lebertransplantation evaluiert werden. Von der Europäischen Fachgesellschaft für Hepatologie sind umfangreiche Behandlungsrichtlinien für Patienten mit

Irina Bergamin · Pamela Meyer-Herbon · Patrizia Künzler-Heule · David Semela

Klinik für Gastroenterologie und Hepatologie, Kantonsspital St. Gallen, St. Gallen, Schweiz

\title{
Klinisches Management von Patienten mit Leberzirrhose
}

Leberzirrhose veröffentlicht worden [2]. Aus diesen Richtlinien und zwei kürzlich erschienenen Übersichtsarbeiten sollen ausgewählte und häufige Aspekte im klinischen Management von Patienten mit Leberzirrhose diskutiert werden [3, 4].

\section{Diagnostik und Behandlung bei Aszites}

Bildung von Aszites ist die häufigste Komplikation bei Patienten mit Leberzirrhose und betrifft jährlich bis zu $10 \%$ bisher kompensierter Zirrhotiker [2]. Bei neu aufgetretenem Aszites oder bei Patienten, welche wegen zunehmendem Aszites hospitalisiert werden, soll eine diagnostische Parazentese durchgeführt werden. Mittels Zellzahl, Differenzierung der Leukozyten, bakteriellen Kulturen und der Albuminkonzentration im Aszites kann die Ursache (portal-hypertensiver Aszites bei Serum-Aszites-AlbuminGradient [SAAG] > 11g/l) und eine allfällige spontan-bakterielle Peritonitis (>250 Neutrophile/ $\mu \mathrm{l})$ diagnostiziert werden. Bei klinischem Verdacht auf eine spontan-bakterielle Peritonitis sollten auch Blutkulturen abgenommen werden. Bei Verdacht auf malignen Aszites ist eine zusätzliche zytologische Analyse sinnvoll.

Unkomplizierter Aszites (keine Infektion, nicht refraktär und ohne hepatorenales Syndrom) kann in der Regel mit Natriumrestriktion (max. 80-120 mmol Natrium resp. max. 4,6-6,9g Kochsalz pro Tag) und Diuretika kontrolliert werden. Spironolacton als AldosteronAntagonist ist bei Aszites das Diuretikum der Wahl: Startdosis $100 \mathrm{mg}$ täglich mit Steigerung der Dosis um $100 \mathrm{mg}$ alle 3 Tage bis maximal $400 \mathrm{mg}$ täglich bei fehlendem Ansprechen $(<2 \mathrm{~kg} \mathrm{Ge}-$ wichtsverlust/Woche). Bei ungenügendem Ansprechen und/oder Hyperkaliämie soll mit einem Schleifendiuretikum (Torasemid oder Furosemid mit $10 \mathrm{mg}$ resp. $40 \mathrm{mg}$ täglich mit Steigerung bis $40 \mathrm{mg}$ resp. $160 \mathrm{mg}$ täglich) ergänzt werden. Diuretika sollen sistiert werden bei schwerer Hyponatriämie (Serum $\mathrm{Na}^{+}$ $<125 \mathrm{mmol} / \mathrm{l}$ ), Niereninsuffizienz resp. hepatorenalem Syndrom, zunehmender hepatischer Enzephalopathie oder bei ausgeprägten Muskelkrämpfen ohne Besserung auf Albumin-Infusionen oder Baclofen (10 mg täglich). Spironolacton muss bei schwerer Hyperkaliämie $\left(\mathrm{K}^{+}>6 \mathrm{mmol} / \mathrm{l}\right)$ sistiert werden;

\begin{tabular}{|ll}
\hline \multicolumn{2}{|l}{ Abkürzungen } \\
\hline$A S A$ & American Society of Anesthesia \\
\hline$B M I$ & Body-Mass-Index \\
\hline COPD & $\begin{array}{l}\text { Chronisch-obstruktive Atemwegser- } \\
\text { krankung }\end{array}$ \\
\hline CT & Computertomographie \\
\hline CTP & Child-Turcotte-Pugh \\
\hline Hb & Hämoglobin \\
\hline$H C C$ & Hepatozelluläres Karzinom \\
\hline$H E$ & Hepatische Encephalopathie \\
\hline$H V P G$ & Hepatic venous pressure gradient \\
\hline MELD & Model of end stage liver disease \\
\hline MRT & Magnetresonanztomographie \\
\hline NSAID & Nichtsteroidale Entzündungshemmer \\
\hline NSBB & Nichtselektiver Betablocker \\
\hline SAAG & Serum-Aszites-Albumin-Gradient \\
\hline SBP & Spontan-bakterielle Peritonitis \\
\hline TIPS & $\begin{array}{l}\text { Transjugulärer portosystemischer } \\
\text { Shunt }\end{array}$ \\
\hline
\end{tabular}


Schleifendiuretika müssen bei schwerer Hypokaliämie $\left(\mathrm{K}^{+}<3 \mathrm{mmol} / \mathrm{l}\right)$ sistiert werden.

Bei Patienten mit gespanntem Aszites soll eine therapeutische Parazentese mit Albuminersatz (8g Albumin i.v. pro Liter drainiertem Aszites) zur Verhinderung einer postinterventionellen zirkulatorischen Dysfunktion erfolgen. Der Nutzen regelmässiger Albumingaben für unkomplizierten Aszites bleibt vorerst noch offen:Die wöchentliche intravenöse Gabe von Albumin ( $40 \mathrm{~g}$ zweimal wöchentlich für 2 Wochen, gefolgt von $40 \mathrm{~g}$ wöchentlich für bis zu 18 Monate) bei Patienten mit unkompliziertem Aszites zeigte in der randomisierten, open-label ANSWERStudie ein verbessertes Überleben (77\% vs. $66 \%$ Kontrollgruppe, $n=440$; [5]). Allerdings konnte eine ähnliche placebokontrollierte, randomisierte Studie keinen Überlebensvorteil zeigen unter intravenöser Albumingabe ( $40 \mathrm{~g}$ alle $2 \mathrm{Wo-}$ chen für 80 Tage) bei 196 für eine Transplantation gelisteten Zirrhosepatienten mit Aszites, weshalb weitere Studien abgewartet werden müssen [6].

Refraktärer Aszites ist definiert als Aszites, welcher mehr als drei therapeutischer Parazentesen bedarf bei eingehaltener Natriumrestriktion und optimal eingestellter diuretischer Medikation (engl. „diuretic-resistant ascites") oder einer limitierten diuretischen Therapie, welche aufgrund von Nebenwirkungen nicht weiter gesteigert werden kann (engl. „diuretic-intractable ascites"). In dieser Situation sind therapeutische Parazentesen mit intravenöser Albuminsubstitution (8g/Liter drainiertem Aszites) indiziert. Diuretika sollen bei refraktärem Aszites sistiert werden, falls die Natriumexkretion unter Diuretika $<30 \mathrm{mmol} /$ Tag beträgt. Zudem sollen auch Betablocker abgesetzt werden, falls der systolische Blutdruck $<90 \mathrm{~mm} \mathrm{Hg}$ beträgt.

Bei refraktärem oder rezidivierendem Aszites nach Ausschöpfung aller oben genannten Behandlungsoptionen kann als alternative Therapie auch ein transjugulärer portosystemischer Shunt (TIPS) erwogen werden. Die Senkung der portalen Hypertonie durch den TIPS-Shunt reduziert die Aszitesbildung, verringert das Risiko einer Varizenblutung und hat sich auch auf das Überleben dieser Patienten positiv ausgewirkt [7]. Dabei müssen Patienten vor einer TIPS-Einlage sorgfältig in Zentren mit Erfahrung evaluiert werden: Die Bilirubinkonzentration im Serum sollte $<50 \mu \mathrm{mol} / \mathrm{l}$ sein, es soll keine mittelgradige oder schwere hepatische Enzephalopathie (> Grad 1 nach Westhaven) vorliegen, und der Patient soll eine gute Herzfunktion haben (Ausschluss einer pulmonalarteriellen Hypertonie, Ausschluss Linksund Rechtsherzinsuffizienz, Ausschluss Aortenklappenstenose). Zudem soll keine aktive Infektion und keine schwere Thrombopenie vorliegen.

Falls ein TIPS nicht möglich ist, können Alternativen wie eine permanente, subkutan tunneliert eingelegte Aszitesdrainage (Pleur $\mathrm{X}^{\circledR}$ Katheter; CareFusion Corporation, San Diego, CA, USA) oder ein operativ eingelegtes Pumpensystem (Alfapump $^{\circledR}$; Sequana Medical AG, Zürich, Schweiz) erwogen werden [8].

\section{Spontan-bakterielle Peritonitis}

Die erhöhte Zahl der Neutrophilen (>250/ $\mu$ l Aszites) ist für die Diagnose einer spontan-bakteriellen Peritonitis (SBP) ausreichend und bedarf keiner positiven Bakterienkultur, da diese trotz vorliegender bakterieller Peritonitis in vielen Fällen $(>50 \%)$ negativ ausfällt. Die Identifikation eines Keimes im Aszites oder in der Blutkultur hilft jedoch, die antibiotische Therapie resistenzgerecht anzupassen. Patienten mit Bakteraszites (definiert durch Neutrophile <250/ $\mu$ l Aszites, bei jedoch positiver bakterieller Kultur) und Zeichen einer Infektion oder systemischen Entzündung sollen antibiotisch behandelt werden [2]. Alternativ sollte die Parazentese wiederholt werden und bei erneut positiven Kulturen unabhängig von der Neutrophilenzahl eine antibiotische Therapie begonnen werden. Analog zur spontan-bakteriellen Peritonitis ist das spontan-bakterielle Pleuraempyem definiert durch positive bakterielle Kulturen und eine erhöhte Neutrophilenzahl (>250/ $\mu$ l Pleuraerguss) oder bei $>500 / \mu$ l bei negativen Kulturen und fehlender Pneumonie [2]. Finden sich in der Asziteskultur multiple Bakterien, spricht dies für eine sekundär bakterielle
Peritonitis (z. B. im Rahmen einer Darmperforation). Diese Patienten sollen rasch mittels CT Abdomen abgeklärt und allenfalls chirurgisch vorgestellt werden. Eine sekundäre bakterielle Peritonitis soll auch differenzialdiagnostisch erwogen werden, wenn die Neutrophilenzahl im Aszites stark erhöht ist, eine hohe Proteinkonzentration im Aszites vorliegt und/ oder wenn der Patient auf eine antibiotische Therapie ungenügend anspricht.

Kann mittels Parazentese eine spontan-bakterielle Peritonitis nachgewiesen werden, soll eine intravenöse antibiotische Therapie gemäss lokaler Erfahrung und Resistenzsituation eingesetzt werden (z. B. Ceftriaxon i.v. 2 g/24h). Zudem verbessert die intravenöse Gabe von Albumin $(1,5 \mathrm{~g} / \mathrm{kg}$ Körpergewicht am Tag 1, $1,0 \mathrm{~g} / \mathrm{kg}$ am Tag 3) das Überleben [2]. Patienten nach behandelter spontan-bakterieller Peritonitis sollen eine langfristige antibiotische Prophylaxe bekommen, wobei primär Norfloxacin (400 mg täglich) empfohlen wird [2]. Nach vollständiger Rückbildung des Aszites und verbesserter Leberfunktion kann das Absetzen von Norfloxacin erwogen werden. Rifaximin $(2 \times 550 \mathrm{mg}$ täglich $)$ als Alternative $\mathrm{zu}$ Norfloxacin wird in Studien geprüft.

\section{Ösophagusvarizen: Screening und Primärprophylaxe}

Patienten mit Leberzirrhose sind durch eine Varizenblutung als weitere wichtige Komplikation gefährdet und sollen deshalb bei Diagnosestellung endoskopisch auf das Vorliegen von Varizen untersucht werden (sog. Varizen-Screening). Falls keine Varizen vorliegen, ist bei kompensierter Leberzirrhose ein nächstes Screening nach 2 oder 3 Jahren empfohlen: nach 2 Jahren bei aktiver Hepatopathie, wie z. B. fortgesetzter Alkoholkonsum oder NASH, nach 3 Jahren bei inaktiver Hepatopathie z. B. bei Abstinenz, behandelter Hepatitis B oder C [9]. Bei dekompensierter Leberzirrhose soll ein jährliches Varizenscreening durchgeführt werden. Gemäss den BAVENOVI-Consensus-Empfehlungen kann bei Patienten mit kompensierter Leberzirrhose mit normaler Thrombozytenzahl $(>150 \mathrm{G} / \mathrm{l})$ und einer transienten Elas- 
Hier steht eine Anzeige.

\section{曾 Springer}


tographie $\left(\right.$ Fibroscan $^{\circledR}$ ) von $<20 \mathrm{kPa}$ auf ein Varizenscreening verzichtet werden, da die Wahrscheinlichkeit, dass behandlungsbedürftige Varizen vorliegen, sehr klein ist $(<5 \%)$ [10]. Kleine (unbehandelte) Ösophagusvarizen $(<5 \mathrm{~mm}$ Durchmesser) sollen nach 1 bis 2 Jahren nachkontrolliert werden.

Falls behandlungsbedürftige Varizen vorliegen, soll eine Primärprophylaxe zur Senkung des Blutungsrisikos durchgeführt werden. Behandlungsbedürftige Ösophagusvarizen sind Varizen mit erhöhter Blutungsgefahr und sind definiert als grosse Varizen ( $>5 \mathrm{~mm}$ Durchmesser) oder kleine Varizen $(<5 \mathrm{~mm}$ Durchmesser) mit mukosalen Risikomerkmalen für eine Blutung (sog. „red spot signs, red wale markings"; [10]). Die Primärprophylaxe soll mit einem nichtselektiven Betablocker (NSBB) oder einer Ligaturbehandlung der Ösophagusvarizen durchgeführt werden. Die Wahl der Primärprophylaxe hängt von der Patientenpräferenz sowie von der Verträglichkeit und Kontraindikationen von NSBB ab (z.B. Herzinsuffizienz NYHA II-IV, AV-Block Grad II-III, Bradykardien <45-50/min, Asthma bronchiale, COPD). Als NSBB werden Propranolol, Carvedilol oder Nadolol empfohlen, wobei in der Schweiz vor allem Propranolol und Carvedilol eingesetzt werden. Propranolol wird zweimal täglich eingenommen; begonnen wird mit 20-40 mg 1-0-1 mit Steigerung alle 2-3 Tage bis zum Erreichen der optimalen Dosis (Ruhepuls 55-60/min, keine orthostatischen Symptome, maximale Tagesdosis $160 \mathrm{mg}$ ). Carvedilol ist ein NSBB mit zusätzlicher alpha-1-Rezeptorblockade und deshalb stärkerem antihypertensivem Effekt. Carvedilol wird mit 6,25 mg einmal täglich initiiert (z.B. abendliche Gabe) und bei guter Verträglichkeit nach 3 Tagen auf 6,25 mg zweimal täglich gesteigert. Carvedilol wird in der Primärprophylaxe und bei kompensierter Leberzirrhose bevorzugt verschrieben. NSBB müssen reduziert oder ausgeschlichen werden falls die systolischen Blutdruckwerte $<90 \mathrm{~mm} \mathrm{Hg}$ betragen. Zudem sollen NSBB sistiert werden, falls eine schwere Hyponatriämie $\left(\mathrm{Na}^{+}<125 \mathrm{mmol} / \mathrm{l}\right)$, eine akute Niereninsuffizienz (z.B. im Rahmen ei-

Schweiz. Gastroenterol. $2021 \cdot 2: 119-127$ https://doi.org/10.1007/s43472-021-00058-8 (c) The Author(s), under exclusive licence to Springer-Verlag GmbH Austria, ein Teil von Springer Nature 2021

\section{Bergamin · P. Meyer-Herbon · P. Künzler-Heule · D. Semela}

\section{Klinisches Management von Patienten mit Leberzirrhose}

\section{Zusammenfassung}

Patientinnen und Patienten mit Leberzirrhose sind durch verschiedene Komplikationen wie Aszites, spontan-bakterielle Peritonitis, Varizenblutung, hepatische Enzephalopathie, hepatorenales Syndrom und hepatozelluläres Karzinom gefährdet. Die Übersichtsarbeit gibt einen Überblick über die Prävention und das klinische Management bei Leberzirrhose basierend auf aktuellen Richtlinien.
Daneben werden Aspekte wie präoperative Risikoabschätzung, Ernährungsempfehlungen, Impfungen und Verschreibung von Medikamenten besprochen.

\section{Schlüsselwörter}

Portale Hypertonie · Aszites · Varizenblutung · Hepatozelluläres Karzinom · Hepatische Encephalopathie

\section{Prise en charge clinique des patients cirrhotiques}

\section{Résumé}

Les patients et patientes atteints de cirrhose du foie sont à risque de subir différentes complications telles qu'ascite, péritonite bactérienne spontanée, saignements variqueux, encéphalopathie hépatique, syndrome hépato-rénal et carcinome hépatocellulaire. Cet article de synthèse donne un aperçu de la prévention et de la prise en charge clinique de la cirrhose du foie sur la base des directrices actuelles. Il discute aussi des aspects tels que l'évaluation préopératoire des risques, les conseils diététiques, les vaccinations et la prescription de médicaments.

\section{Mots clés}

Hypertension portale $\cdot$ Ascite $\cdot$ Saignement des varices - Carcinome hépatocellulaire. Encéphalopathie hépatique

\section{Gestione clinica dei pazienti con cirrosi epatica}

\section{Riassunto}

I pazienti con cirrosi epatica rischiano svariate complicanze come ascite, peritonite batterica spontanea, emorragia variceale, encefalopatia epatica, sindrome epatorenale e carcinoma epatocellulare. La rassegna offre una panoramica sulla prevenzione e la gestione clinica della cirrosi epatica sulla base delle direttive attuali. Inoltre, vengono discussi aspetti come la valutazione del rischio preoperatorio, le raccomandazioni dietetiche, le vaccinazioni e la prescrizione di farmaci.

\section{Parole chiave}

Ipertensione portale $\cdot$ Ascite $\cdot$ Emorragia variceal · Carcinoma epatocellulare Encefalopatia epatica nes hepatorenalen Syndroms) oder eine spontan-bakterielle Peritonitis auftreten. Tritt Aszites unter Carvedilol auf, kann ein Wechsel auf Propranolol versucht werden. Aktuelle Richtlinien empfehlen keine endoskopischen Varizenkontrollen unter installierter Primärprophylaxe mit NSBB. Können NSBB im Rahmen der Primärprophylaxe nicht toleriert werden, sollen behandlungsbedürftige Varizen mittels Ligaturbehandlung eradiziert werden.

\section{Management bei Varizen- blutung und Sekundär- prophylaxe}

Varizenblutungen machen $70 \%$ aller oberen gastrointestinalen Blutungen bei Patienten mit portaler Hypertonie aus. Zum detaillierten Management der akuten Varizenblutungen wird auf die Europäischen Richtlinien und eine aktuelle Übersichtsarbeit verwiesen [2, 11]. Zusammengefasst soll bei Verdacht auf eine akute Varizenblutung eine notfallmässige Hospitalisierung erfolgen und eine intravenöse vasoaktive Therapie 
zur Drucksenkung der portalen Hypertonie begonnen werden (für 3-5 Tage Terlipressin [ $2 \mathrm{mg}$ i.v. alle $4 \mathrm{~h}$ für $48 \mathrm{~h}$, danach $1 \mathrm{mg}$ alle $4 \mathrm{~h}$ ], Octreotid [initialer i.v. Bolus $50 \mu \mathrm{g}$ gefolgt von einer kontinuierlichen Infusion mit $50 \mu \mathrm{g} / \mathrm{h}$ ] oder Somatostatin [initialer i.v. Bolus $250 \mu \mathrm{g}$ gefolgt von einer kontinuierlichen Infusion mit $250 \mu \mathrm{g} / \mathrm{h}$, welche bis $500 \mu \mathrm{g} / \mathrm{h}$ gesteigert werden kann; in der Schweiz für Varizenblutungen jedoch nicht zugelassen]). Zudem soll der Patient nach hämodynamischer Stabilisierung innert $12 \mathrm{~h}$ einer oberen Endoskopie zugeführt werden. Vor der Endoskopie kann zur Verbesserung der Übersicht eine Magenentleerung mit Erythromycin induziert werden (250 mg i.v. 30-120 min vor der Endoskopie; Cave Kontraindikation verlängerte QT-Zeit im EKG). Bei nachgewiesener Varizenblutung soll in der gleichen Sitzung eine Ligaturbehandlung der Varizen erfolgen. Gelingt es trotz der genannten Massnahmen nicht, die Blutung zu stillen, soll die Einlage eines Notfall-TIPS (sog. rescueTIPS) evaluiert werden. Ist ein solcher Notfall-TIPS nicht verfügbar, kann zur kurzfristigen Blutstillung und Überbrückung eine Ballon-Tamponade oder ein Ösophagusstent eingelegt werden. Ein TIPS soll auch nach erfolgreich gestillter Blutung innert $72 \mathrm{~h}$ im Rahmen einer Sekundärprophylaxe evaluiert werden (sog. „early pre-emptive“ TIPS): bei Hochrisikopatienten (Child-TurcottePugh[CTP]-Score C mit 10 bis 13 Punkten) konnte durch diese Massnahme ein verbessertes Überleben durch Verhinderung einer Rezidivblutung gezeigt werden. Bei CTP-C-Patienten mit 14 bis 15 Punkten soll wegen der sehr eingeschränkten Leberfunktion und dem drohenden Leberversagen keine TIPSEinlage erfolgen. Bei CTP-B-Patienten nach aktiver Varizenblutung konnte eine TIPS-Einlage das Risiko einer Rezidivblutung zwar senken, hatte jedoch keinen Effekt auf das Überleben, weshalb in dieser Gruppe noch keine definitiven Empfehlungen vorliegen.

Bluttransfusionen bei Patienten mit Varizenblutung sollen restriktiv gehandhabt werden, um den Druck in den Varizen nicht zu erhöhen und die Blutung nicht weiter zu unterhalten. Dabei soll ein
Hämoglobin-Zielbereich von 70-90 g/l angestrebt werden und die Transfusionsgrenze (abhängig von kardiovaskulären Vorerkrankungen) idealerweise bei $70 \mathrm{~g} / 1$ angesetzt werden. Zur Prophylaxe einer spontan-bakteriellen Peritonitis nach Varizenblutung soll früh eine antibiotische Behandlung initiiert werden (z.B. Ceftriaxon i.v. $1 \mathrm{~g} /$ Tag für bis zu 7 Tage, alternativ Norfloxacin p.o. $400 \mathrm{mg}$ 1-01). Auf NSBB sollte während einer akuten Varizenblutung verzichtet werden. Nichtselektive Betablocker sollen jedoch nach Stabilisierung im Rahmen einer Sekundärprophylaxe eingesetzt werden und die Varizen im Verlauf bis zur Eradikation mit Ligaturen behandelt werden. Falls ein „early pre-emptive“ TIPS zur Sekundärprophylaxe eingesetzt wurde und die portale Hypertonie dadurch korrigiert ist, kann der NSBB abgesetzt werden, und es braucht in der Regel (bei kollabierten Ösophagusvarizen) keine Ligaturbehandlungen mehr.

\section{Hepatische Enzephalopathie}

Die hepatische Enzephalopathie (HE) ist eine potenziell reversible Störung der Funktion des Zentralnervensystems bei Leberinsuffizienz und/oder bei portosystemischen Shunts, welche sich mit einem breiten Spektrum von Symptomen manifestieren kann (z.B. kognitive Dysfunktion mit Gedächtnisstörungen, Schlafstörungen, „flapping tremor", Konfusion, Koma). Die Erkennung und Behandlung der auslösenden Faktoren (z.B. Exsikkose, Obstipation, Infektion, gastrointestinale Blutung, Opiate, Sedativa) und der Einsatz von nichtabsorbierbaren Disacchariden (z. B. Lactitol oder Lactulose 30-50 ml dreimal täglich mit dem Ziel von 2-3 weichen Stuhlgängen täglich) sind zentral. Bei schwerer HE mit Vigilanzminderung können diese Medikamente über eine Magensonde und allenfalls zusätzlich als Einlauf (z. B. $300 \mathrm{ml}$ Lactulose in $700 \mathrm{ml}$ Wasser) verabreicht werden. Zur Verminderung des Wiederauftretens von Episoden einer manifesten $\mathrm{HE}$ oder bei rekurrenter HE trotz Einsatz von Lactulose oder Lactitol haben mehrere Studien den Nutzen einer zusätzlichen langfristigen Gabe von Rifaximin (Tbl.
550 mg 1-0-1) belegen können [12]. Dabei reduziert dieses orale und minimal absorbierte Antibiotikum die Produktion und Absorption von intestinalem Ammoniak. Bei Patienten mit HE sollen Opiate und Sedativa gemieden werden. Eine Restriktion der Eiweisszufuhr wegen HE wird nicht empfohlen. Patienten mit HE haben eine eingeschränkte Fahrtüchtigkeit und dürfen kein Fahrzeug lenken.

\section{Seltenere Komplikationen der portalen Hypertonie}

Auch auf seltenere Komplikationen der portalen Hypertonie wie das hepatorenale Syndrom, die portopulmonale Hypertonie und das hepatopulmonale Syndrom soll geachtet werden. Bezüglich detaillierter Diagnostik und Therapie dieser Entitäten wird auf die aktuellen Europäischen Richtlinien verwiesen [2]. Patienten mit Leberzirrhose und erniedrigter Sauerstoffsättigung, Zyanose oder Trommelschlegelfinger sollen mittels Kontrastmittel-Echokardiographie oder einer Lungenszintigraphie auf das Vorliegen eines hepatopulmonalen Syndroms mit intrapulmonaler Gefässdilatation und vaskulärem Shunting untersucht werden. Beim hepatopulmonalen Syndrom ist eine Lebertransplantation die einzige kurative Therapie. Bei Zeichen einer Rechtsherzbelastung muss differenzialdiagnostisch an eine portopulmonale Hypertonie (pulmonalarterielle Hypertonie bei bestehender portaler Hypertonie) gedacht werden und der Patient echokardiographisch und mittels Rechtsherzkatheter abgeklärt werden. Auch vor Einlage eines TIPS oder einer Listung zur Lebertransplantation muss eine portopulmonale Hypertonie ausgeschlossen werden. Bei nachgewiesener portopulmonaler Hypertonie kann eine Therapie mit dem EndothelinRezeptorantagonisten Macitentan interdisziplinär (Kardiologie, Pneumologie) evaluiert werden.

\section{Surveillance für hepato- zelluläres Karzinom}

Je nach hepatologischer Grunderkrankung entwickeln jährlich 1-8\% der Pati- 
enten mit Leberzirrhose neu ein hepatozelluläres Karzinom (HCC). Eine kurative Therapie des HCC, wie z. B. eine Resektion, Ablation oder Transplantation, ist nur im Frühstadium möglich, weshalb ein regelmässiges Screening alle 6 Monate (sog. HCC-Surveillance) mittels Sonographie bei Patienten mit Leberzirrhose empfohlen ist [13]. Die Sonographiekann zur Verbesserung der Sensitivität mit einer Bestimmung des Tumormarkers alpha-Fetoprotein (AFP) ergänzt werden. Bei eingeschränkten Schallbedingungen wird ein 4-Phasen-CT oder eine MRT mit Kontrastmittel der Leber empfohlen. Jede neue, unklare oder grössenprogrediente fokale Leberläsion bei Patienten mit Leberzirrhose muss mittels MRT, CT und/ oder Biopsie abgeklärt werden [13]. Wegen erhöhter Blutungsgefahr und potenziellem Tumorseeding sollen Rundherdbiopsien (idealerweise kombiniert mit einer zusätzlichen Biopsie des Leberparenchyms) nur durch erfahrene Spezialisten und mittels Übernadel erfolgen. Bei nachgewiesenem HCC oder unklarer fokaler Leberläsion sollen Patienten an einem Zentrum mit interdisziplinärem Tumorboard vorgestellt werden.

\section{Präoperatives Risiko- Assessment}

Patienten mit Leberzirrhose haben insbesondere bei bestehender portaler Hypertonie eine deutlich erhöhte perioperative Morbidität und Mortalität infolge Auftretens von postoperativen Komplikationen (z. B. Aszites, [Varizen-]Blutungen, Infektionen, Nierenversagen) sowie Acute-on-chronic-Leberversagen. Deshalb müssen Patienten mit Leberzirrhose vor einem geplanten Eingriff eingehend abgeklärt und der Nutzen des Eingriffs zum Risiko einer postoperativen hepatischen Dekompensation interdisziplinär (Chirurgie, Anästhesie, Hepatologie) kritisch abgewogen werden. Der kürzlich entwickelte VOCAL-Penn-Score (www. vocalpennscore.com) oder der MELDScore in Kombination mit dem Alter und ASA-Klasse des Patienten (https://www. mayoclinic.org/medical-professionals/ transplant-medicine/calculators/postoperative-mortality-risk-in-patientswith-cirrhosis/itt-20434721) sind ge- eignet, um die postoperative Mortalität bei Patienten mit Leberzirrhose vorgängig einzuschätzen [14, 15]. Wegen der erhöhten Mortalität sollen präoperativ verschiedene Aspekte und Alternativen zur Operation berücksichtigt werden [16]: Ist die geplante Operation verschiebbar oder zwingend notwendig (z.B. onkologische Indikation)? Ist eine konservative Therapieoption oder ein minimal-invasiver Eingriff möglich (z.B. Endoskopie, Laparoskopie)? Kann die Leberfunktion vor der Operation verbessert werden (z.B. Behandlung der hepatologischen Grunderkrankung, Alkoholabstinenz)? Ist allenfalls ein prophylaktischer, präoperativ eingelegter TIPS bei ausgeprägter portaler Hypertonie sinnvoll (HVPG > $16 \mathrm{~mm} \mathrm{Hg}$ )? Liegt eine dekompensierte Leberzirrhose vor und muss der Patient vorgängig lebertransplantiert oder vorsorglich für eine allfällige Lebertransplantation gelistet werden?

\section{Listung zur Lebertransplantation}

Bei hepatischer Dekompensation sinkt die Lebenserwartung von Patienten mit Leberzirrhose von durchschnittlich 12 auf 2 Jahre [2]. Aufgrund dieser deutlich verkürzten Lebenserwartung sollte beim Auftreten von Zirrhose-assoziierten Komplikationen oder einer Verschlechterung der Leberfunktion mit einem MELD-Score $\geq 15$ Punkten in Absprache mit einem Transplantationszentrum (Universitätsspitäler Bern, Genf, Zürich) eine Evaluation für eine Listung zur Lebertransplantation erfolgen. Auch Patienten mit einem hepatozellulären Karzinom im Frühstadium sollen rechtzeitig für eine Listung zur Lebertransplantation evaluiert werden [13]. Indikationen, Kontraindikationen und Transplantationsabklärungen in der Schweiz bei Patienten mit Leberzirrhose sind in einer kürzlich erschienenen Übersichtsarbeit beschrieben [17].

\section{Pflegerische Betreuung von Patienten mit Leberzirrhose}

Spezialisiertes Pflegepersonal kann einen wichtigen Beitrag in der Betreuung und
Schulung von Zirrhose-Patienten und deren Familien leisten. Insbesondere Themen wie z.B. Früherkennung von Komplikationen (Auftreten von hepatischer Enzephalopathie, Zunahme von Aszites), zuverlässige Medikamenteneinnahme, Motivation zur körperlichen Betätigung, gesunde Ernährung sowie Meiden von Übergewicht, Alkohol und Nikotin sollten regelmässig besprochen werden [3, 18, 19]. Schulung kann Dekompensationen und stationäre Aufnahmen von Patienten mit Leberzirrhose reduzieren [4]. Die Schweizerische Vereinigung für Hepatologie (SASL) hat eine durch Pflegeexpertinnen produzierte Broschüre zur Patientenschulung bei Leberzirrhose bereitgestellt (informationlebererkrankung@kssg.ch).

\section{Impfungen bei Leberzirrhose}

Schutz vor Infektionen durch Impfungen kann Dekompensationen bei Patienten mit Leberzirrhose verhindern. Gemäss Schweizerischem Impfplan 2021 sollen Personen mit chronischen Lebererkrankungen und insbesondere mit Leberzirrhose gegen Hepatitis A (2 Dosen im Abstand von 6 Monaten) und Hepatitis B (3 Dosen bei Monaten 0, 1 und 6) geimpft werden. Eine Immunisierung gegen Hepatitis B ist auch gegen das Hepatitis-Delta-Virus protektiv. Zudem wird die jährliche Impfung gegen Influenza und die einmalige Impfung gegen Pneumokokken empfohlen. Eine Impfung gegen das Hepatitis-E-Virus als häufigste Ursache einer akuten viralen Hepatitis und als beschriebenen Auslöser eines Acute-on-chronic-Leberversagens bei Patienten mit Leberzirrhose ist bisher in Europa nicht erhältlich.

Mehrere Studien haben gezeigt, dass Patienten mit Leberzirrhose eine deutlich erhöhte Morbidität und Mortalität bei COVID-19 aufweisen, weshalb eine Immunisierung gegen das SARS-CoV2-Virus insbesondere bei Leberzirrhose klar empfohlen ist. Patienten unter immunsuppressiver Therapie (Mycophenolat-Mofetil, Tacrolimus, Ciclosporin, Prednison $>20 \mathrm{mg}$ täglich, Rituximab oder unter kombinierter Immunsuppression), z. B. bei Autoimmunhepatitis, Overlap-Syndrom oder nach Leber- 
Hier steht eine Anzeige.

\section{曾 Springer}


transplantation, sollen bei ungenügenden Impftitern einen Monat nach der zweiten SARS-CoV-2-Impfung (IgGAntikörper gegen die S1-Domäne des Spike-Proteins von SARS-CoV-2) eine zusätzliche dritte Impfdosis erhalten. Auffrischimpfungen für Patienten mit Leberzirrhose als besonders gefährdete Personen sollen gemäss den Empfehlungen des Bundesamts für Gesundheit durchgeführt werden.

\section{Ernährungsempfehlungen}

Patienten mit Leberzirrhose haben ein erhöhtes Risiko für Malnutrition, Sarkopenie und Kachexie, weshalb ein Assessment der Ernährung und eine entsprechende Beratung zentral sind [19]. Zur Erhaltung der Muskelmasse soll auf eine ausgewogene Ernährung mit genügender Eiweisszufuhr $(1,2-1,5 \mathrm{~g} / \mathrm{kg}$ Körpergewicht) geachtet werden (optimal in Kombination mit regelmässiger körperlicher Betätigung; [18, 19]). Die tägliche Energiezufuhr bei Patienten ohne Adipositas soll $35 \mathrm{kcal} / \mathrm{kg}$ Körpergewicht erreichen. Idealerweise werden kleinere, aber häufige hochkalorische Mahlzeiten inklusive eines Abendsnacks vor dem Schlafengehen eingenommen (z.B. 3 Hauptmahlzeiten, 3 Zwischenmahlzeiten). Bei Patienten mit Adipositas $\left(\mathrm{BMI}>30 \mathrm{~kg} / \mathrm{m}^{2}\right.$, korrigiert für Wasserretention und Ödeme) soll ein langsamer Gewichtsverlust von $>5$ bis $10 \%$ angestrebt werden durch vermehrte körperliche Aktivität und Reduktion der täglichen Kalorieneinnahme um $500-800 \mathrm{kcal}$ bei genügender Proteinzufuhr (>1,5g Protein/kg Körpergewicht täglich; [19]). Eine entsprechende Ernährungsberatung des Patienten und seiner Familie ist sinnvoll. Längeres Fasten soll vermieden werden. Auf Süssgetränke mit hohem Gehalt an Fruktose soll insbesondere bei nichtalkoholischer Fettlebererkrankung verzichtet werden. Bei nachgewiesenem Mangel soll auf genügende Supplementation von Vitaminen, Eisen und Zink geachtet werden. Insbesondere findet sich bei Patienten mit Leberzirrhose häufig ein VitaminD-Mangel, welcher substituiert werden soll. Bei Aszites soll eine Natriumrestriktion (max. 80-120 mmol Natrium resp. max. 4,6-6,9g Kochsalz pro Tag) eingehalten werden, was in der Regel durch Meiden von Nachsalzen und Verzicht auf Fertigprodukte erreicht werden kann. Von Diäten mit extrem tiefer $\mathrm{Na}$ triumzufuhr $(<40 \mathrm{mmol} \mathrm{Na} / \mathrm{Tag})$ wird wegen vermehrten Diuretika-assoziierten Komplikationen abgeraten [2]. Die Trinkmenge resp. Flüssigkeitszufuhr soll nur bei schwerer Hyponatriämie $\left(\mathrm{Na}^{+}\right.$ $<120 \mathrm{mmol} / \mathrm{l}$ ) eingeschränkt werden [4].

Auf Alkohol (und Nikotin) soll gänzlich verzichtet werden. Mehrere klinische und epidemiologische Studien konnten hingegen für Kaffee einen hepatoprotektiven Effekt zeigen, wobei regelmässige Konsumation von Kaffee (ideal 2-4 Tassen täglich) günstige Effekte auf Steatose, Fibrose und Karzinogenese der Leber zu haben scheint [20].

\section{Einsatz von Medikamenten bei Leberzirrhose}

Der Einsatz jedes Medikamentes bei Patienten mit Leberzirrhose muss wegen potenzieller Hepatotoxizität, $\mathrm{Ne}$ benwirkungen und Dosisanpassungen aufgrund verzögerter Elimination insbesondere bei CTP Stadium B und C wohlüberlegt sein [21]. Die Leberwerte und die Leberfunktion sollen bei Einsatz von neuen Medikamenten regelmässig kontrolliert werden. Gewisse Medikamente sollten nach Möglichkeit gemieden oder überhaupt nicht eingesetzt werden. So sind nichtsteroidale Entzündungshemmer (NSAID) bei Patienten mit Leberzirrhose kontraindiziert wegen der Gefahr von gastrointestinalen Blutungen, einer akuten Niereninsuffizienz mit vermehrter Natriumretention und Hyponatriämie [2, 4]. Ausgenommen von dieser Kontraindikation ist niedrig dosiertes Aspirin (100 mg) im Rahmen einer kardiovaskulären Prophylaxe. Geeignete Analgetika bei Patienten mit Leberzirrhose sind Paracetamol bis $2 \mathrm{~g}$ täglich (unter Meidung von Alkohol und ohne eine Acute-on-chronic-Hepatopathie) und niedrig dosiertes Tramadol. Bei hepatischer Encephalopathie sollen Opiate und Sedativa gemieden werden. In Patienten mit alkoholischer Hepatitis oder Zirrhose mit Alkoholentzugserscheinungen sollen zur Vermeidung einer Übersedierung nur Benzodiazepine mit kurzer Halbwertszeit verabreicht werden (z. B. Lorazepam, Oxazepam; [4]). Bei Insomnie kann der Einsatz des Serotonin-Wiederaufnahmehemmers Trazodon (abendliche Gabe von $100 \mathrm{mg}$ ) erwogen werden [4]. ACE-Hemmer und AT-II-Antagonisten sollten bei Patienten mit Aszites wegen Hypotonie und möglicher Niereninsuffizienz nicht eingesetzt werden. Auch Aminoglykoside sollten aus dem gleichen Grund nicht oder nur sehr restriktiv eingesetzt werden. Der Einsatz und die Indikation von Protonenpumpen-Hemmern soll regelmässig überprüft werden, da vermehrte Infekte bei Patienten unter dieser Therapie beobachtet wurden [4].

Unter Medikamenten mit bekannter Hepatotoxizität (z. B. Amiodaron, Methotrexat, Tamoxifen, 5- Fluorouracil, Irinotecan) müssen engmaschige Kontrollen erfolgen. Vor dem Einsatz von Rituximab muss wegen bekannter schwerer viraler Reaktivierung eine aktive oder durchgemachte Hepatitis B ausgeschlossen resp. eine Prophylaxe mit Tenofovir oder Entecavir installiert werden (Dauer bis mindestens 18 Monate über die letzte Gabe von Rituximab).

Der Einsatz von Statinen bei gegebener Indikation wird als relativ sicher angesehen (z. B. Simvastatin mit Startdosis $20 \mathrm{mg}$ und Steigerung auf $40 \mathrm{mg}$ nach 1-2 Wochen). Statine haben in mehreren Studien und Metaanalysen einen günstigen Effekt auf die portale Hypertonie, Dekompensation und Mortalität bei Patienten mit Leberzirrhose gezeigt [4]. Wegen der Gefahr einer Rhabdomyolyse unter Statinen soll die Creatin-Kinase regelmässig bestimmt und auf Muskelschmerzen geachtet werden. Neben Statinen haben auch NSBB in der prospektiven PREDESCI-Studie einen günstigen Effekt auf Dekompensation bei Patienten mit Leberzirrhose und klinisch signifikanter portaler Hypertonie (kleine Ösophagusvarizen oder $\mathrm{HVPG} \geq 10 \mathrm{~mm} \mathrm{Hg}$ ) gezeigt [22]. NSBB sollen sistiert werden, falls eine schwere Hyponatriämie $\left(\mathrm{Na}^{+}\right.$ $<125 \mathrm{mmol} / \mathrm{l}$ ), eine akute Niereninsuffizienz (z. B. im Rahmen eines hepatorenalen Syndroms) oder eine spontan-bakterielle Peritonitis auftreten. Ein Eisenmangel, z. B. im Rahmen einer portal-hyper- 
tensiven Gastropathie bei Patienten mit Leberzirrhose, soll mit i.v. Eisensubstitution (Eisencarboxymaltose) behoben werden; auf beschriebene Hypophosphatämien soll dabei geachtet werden.

\section{Take-Home Message \\ Patientinnen und Patienten mit Le- berzirrhose haben infolge portaler Hypertonie und des Risikos für hepa- tozelluläre Karzinome eine deutlich erhöhte Morbidität und Mortalität. Re- gelmässige klinische Betreuung mit Vorsorgeuntersuchungen durch den Grundversorger, Gastroenterologen und Hepatologen sowie Schulung der Betroffenen sollen deshalb schwerwie- gende Komplikationen verhindern und eine frühzeitige Diagnose bei Dekom- pensation erlauben. Die Indikation für Operationen und der Einsatz von Medi- kamenten müssen bei bekannter Leber- zirrhose kritisch gestellt werden. Patien- ten mit dekompensierter Leberzirrhose oder einem hepatozellulären Karzinom im Frühstadium sollen rechtzeitig für eine Listung zur Lebertransplantation evaluiert werden.}

\section{Korrespondenzadresse}

PD Dr. med. Dr. phil. nat. David Semela Klinik für Gastroenterologie und Hepatologie, Kantonsspital St. Gallen

Rorschacherstraße 95, 9007 St. Gallen, Schweiz david.semela@kssg.ch

\section{Einhaltung ethischer Richtlinien}

Interessenkonflikt. I. Bergamin, P. Meyer-Herbon, P. Künzler-Heule und D. Semela geben an, dass kein Interessenkonflikt besteht.

Für diesen Beitrag wurden von den Autoren keine Studien an Menschen oder Tieren durchgeführt. Für die aufgeführten Studien gelten die jeweils dort angegebenen ethischen Richtlinien.

\section{Literatur}

1. Pimpin $L$, Cortez-Pinto $H$, Negro $F$, Corbould $E$, Lazarus JV, Webber L, Sheron N, Steering Committee EASLHEPAHEALTH (2018) Burden of liver disease in Europe: Epidemiology and analysis of risk factors to identify prevention policies. J Hepatol 69:718-735. https://doi.org/10.1016/j. jhep.2018.05.011
2. Angeli $P$, Bernardi M, Villanueva $C$, Francoz $C$, Mookerjee RP, Trebicka J, Krag A, Laleman W, Gines P (2018) EASL Clinical Practice Guidelines for the management of patients with decompensated cirrhosis. J Hepatol 69:406-460. https://doi.org/ 10.1016/j.jhep.2018.03.024

3. Gines $P$, Krag A, Abraldes JG, Sola E, Fabrellas N, Kamath PS (2021) Liver cirrhosis. Lancet 398:1359-1376. https://doi.org/10.1016/S01406736(21)01374-X

4. Ge PS, Runyon BA (2016) Treatment of patients with cirrhosis. N Engl J Med 375:767-777. https:// doi.org/10.1056/NEJMra1504367

5. Caraceni P, Riggio O, Angeli P et al (2018) Longterm albumin administration in decompensated cirrhosis (ANSWER): an open-label randomised trial. Lancet 391:2417-2429. https://doi.org/10. 1016/S0140-6736(18)30840-7

6. Sola E, Sole C, Macarena ST et al (2018) Midodrine and albumin for prevention of complications of cirrhosis in patients in the waiting list for liver transplantation. A randomized, multicenter, double-blind, placebo-controlled trial. J Hepatol 69:1250-1259. https://doi.org/10.1016/j.jhep. 2018.08.006

7. Bureau C, Thabut D, Oberti F et al (2017) Transjugular intrahepatic portosystemic shunts with covered stents increase transplant-free survival of patients with cirrhosis and recurren ascites. Gastroenterology 152:157-163. https:// doi.org/10.1053/j.gastro.2016.09.016

8. Bureau C, Adebayo D, Chalret de Rieu Metal (2017) Alfapump system vs. Large volume paracentesis for refractory ascites: a multicenter randomized controlled study. J Hepatol 67:940-949. https:// doi.org/10.1016/j.jhep.2017.06.010

9. Garcia-Tsao G, Abraldes JG, Berzigotti A, Bosch J (2016) Portal hypertensive bleeding in cirrhosis: Risk stratification, diagnosis, and management 2016 practice guidance by the American Association for the study of liver diseases. Hepatology 65:310-335. https://doi.org/10.1002/hep.28906

10. de Franchis R, Faculty BVI (2015) Expanding consensus in portal hypertension: report of the Baveno VI Consensus Workshop: Stratifying risk and individualizing care for portal hypertension. J Hepatol 63:743-752. https://doi.org/10.1016/j. jhep.2015.05.022

11. Pfisterer N, Ungerer LW, Reiberger T (2021) Clinical algorithms for the prevention of variceal bleeding and rebleeding in patients with liver cirrhosis. World J Hepatol 13:731-746. https://doi.org/10. 4254/wjh.v13.i7.731

12. Gluud LL, Vilstrup H, Morgan MY (2016) Nonabsorbable disaccharides versus placebo/no intervention and lactulose versus lactitol for the prevention and treatment of hepatic encephalopathy in people with cirrhosis. Cochrane Database Syst Rev. https://doi.org/10.1002/14651858. CD003044.pub3

13. Goossens N, Toso C, Heim MH (2020) Management of hepatocellular carcinoma: SASL expert opinion statement. Swiss Med Wkly 150:1-7. https://doi. org/10.4414/smw.2020.20296

14. Mahmud N, Fricker Z, Hubbard RA, loannou GN Lewis JD, Taddei TH, Rothstein KD, Serper M, Goldberg DS, Kaplan DE (2021) Risk prediction models for post-operative mortality in patients with cirrhosis. Hepatology 73:204-218. https:// doi.org/10.1002/hep.31558

15. Teh SH, Nagorney DM, Stevens SR, Offord KP, Therneau TM, Plevak DJ, Talwalkar JA, Kim WR, Kamath PS (2007) Risk factors for mortality after surgery in patients with cirrhosis. Gastoenterology
132:1261-1269. https://doi.org/10.1053/j.gastro. 2007.01.040

16. Northup PG, Friedman LS, Kamath PS (2019) AGA Clinical Practice Update on surgical risk assessment and perioperative management in cirrhosis: expert review. Clin Gastroenterol Hepatol 17:595-606. https://doi.org/10.1016/j.cgh.2018.09.043

17. Staufer K, Galante A, De Gottardi A (2020) Lebertransplantation in der Schweiz 2020. Schweiz Gastroenterol 1:113-120. https://doi.org/10. 1007/s43472-020-00025-9

18. Tandon P, Ismond KP, Riess Ket al (2018) Exercise in cirrhosis: Translating evidence and experience to practice. J Hepatol 69:1164-1177. https://doi.org/ 10.1016/j.jhep.2018.06.017

19. Merli M, Berzigotti A, Zelber-Sagi S, Dasarathy S, Montagnese S, Genton L, Plauth M, Pares A (2019) EASL Clinical Practice Guidelines on nutrition in chronic liver disease. J Hepatol 70:172-193. https://doi.org/10.1016/j.jhep.2018.06.024

20. Alferink LJM, Kiefte-de JJC, Murad SD (2018) Potential mechanisms underlying the role of coffee in liver health. Semin Liver Dis 38:193-214. https:// doi.org/10.1055/s-0038-1666869

21. Andrade RJ, Aithal GP, Björnsson ES, Kaplowitz N, Kullak-Ublick GA, Larrey D, Karlsen TH (2019) EASL clinical practice guidelines: drug-induced liver injury. J Hepatol 70:1222-1261. https://doi.org/ 10.1016/j.jhep.2019.02.014

22. Villanueva C, Albillos A, Genescà J (2019) Beta blockers to prevent decompensation of cirrhosis in patients with clinically significant portal hypertension (PREDESCI): a randomised, doubleblind, placebo-controlled, multicentre trial. Lancet 393:1597-1608. https://doi.org/10.1016/S01406736(18)31875-0

Hinweis des Verlags. Der Verlag bleibt in Hinblick auf geografische Zuordnungen und Gebietsbezeichnungen in veröffentlichten Karten und Institutsadressen neutral. 